\title{
Prevalence of germline $B R C A$ mutations in HER2-negative metastatic breast cancer: global results from the real-world, observational BREAKOUT study
}

Joyce O'Shaughnessy ${ }^{1 *}$, Christine Brezden-Masley², Marina Cazzaniga ${ }^{3}$, Tapashi Dalvi ${ }^{4}$, Graham Walker ${ }^{5}$, James Bennett ${ }^{5}$ and Shozo Ohsumi ${ }^{6}$

\begin{abstract}
Background: The global observational BREAKOUT study investigated germline BRCA mutation (gBRCAm) prevalence in a population of patients with human epidermal growth factor receptor 2 (HER2)-negative metastatic breast cancer (MBC).

Methods: Eligible patients had initiated first-line cytotoxic chemotherapy for HER2-negative MBC within 90 days prior to enrollment. Hormone receptor (HR)-positive patients had experienced disease progression on or after prior endocrine therapy, or endocrine therapy was considered unsuitable. gBRCAm status was determined using baseline blood samples or prior germline test results. For patients with a negative gBRCAm test, archival tissue was tested for somatic BRCAm and homologous recombination repair mutations (HRRm). Details of first-line cytotoxic chemotherapy were also collected.

Results: Between March 2017 and April 2018, 384 patients from 14 countries were screened and consented to study enrollment; 341 patients were included in the full analysis set (median [range] age at enrollment: 56 [25-89] years; 256 (75.3\%) postmenopausal). Overall, 33 patients (9.7\%) had a gBRCAm (16 [4.7\%] in gBRCA1 only, 12 [3.5\%] in gBRCA2 only, and 5 [1.5\%] in both gBRCA1 and gBRCA2). gBRCAm prevalence was similar in HR-positive and HRnegative patients. gBRCAm prevalence was $9.0 \%$ in European patients and $10.6 \%$ in Asian patients and was higher in patients aged $\leq 50$ years at initial breast cancer (BC) diagnosis (12.9\%) than patients aged $>50$ years (5.4\%). In patients with any risk factor for having a gBRCAm (family history of BC and/or ovarian cancer, aged $\leq 50$ years at initial $B C$ diagnosis, or triple-negative $B C$ ), prevalence was $10.4 \%$, versus $5.8 \%$ in patients without these risk factors. HRRm prevalence was $14.1 \%(n=9 / 64)$ in patients with germline BRCA wildtype.

\footnotetext{
* Correspondence: Joyce.OShaughnessy@usoncology.com

'Baylor University Medical Center, Texas Oncology and US Oncology, Dallas,

TX, USA

Full list of author information is available at the end of the article
}

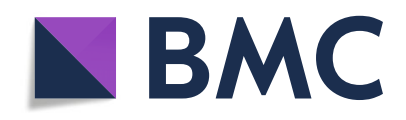

(- The Author(s). 2020 Open Access This article is licensed under a Creative Commons Attribution 4.0 International License, which permits use, sharing, adaptation, distribution and reproduction in any medium or format, as long as you give appropriate credit to the original author(s) and the source, provide a link to the Creative Commons licence, and indicate if changes were made. The images or other third party material in this article are included in the article's Creative Commons licence, unless indicated otherwise in a credit line to the material. If material is not included in the article's Creative Commons licence and your intended use is not permitted by statutory regulation or exceeds the permitted use, you will need to obtain permission directly from the copyright holder. To view a copy of this licence, visit http://creativecommons.org/licenses/by/4.0/. The Creative Commons Public Domain Dedication waiver (http://creativecommons.org/publicdomain/zero/1.0/) applies to the data made available in this article, unless otherwise stated in a credit line to the data. 
(Continued from previous page)

Conclusions: Patient demographic and disease characteristics supported the association of a gBRCAm with younger age at initial BC diagnosis and family history of BC and/or ovarian cancer. gBRCAm prevalence in this cohort, not selected on the basis of risk factors for gBRCAm, was slightly higher than previous results suggested. gBRCAm prevalence among patients without a traditional risk factor for harboring a gBRCAm (5.8\%) supports current guideline recommendations of routine gBRCAm testing in HER2-negative MBC, as these patients may benefit from poly(ADP-ribose) polymerase (PARP) inhibitor therapy.

Trial registration: NCT03078036.

Keywords: Breast cancer susceptibility genes, BRCA, Prevalence, Observational

\section{Background}

Breast cancer is the most commonly diagnosed cancer in women (excluding non-melanoma skin cancers) and is the most common cause of cancer deaths in women worldwide [1]. The breast cancer susceptibility genes (BRCA1 and BRCA2) encode proteins critically involved in the repair of DNA double-strand breaks [2]. A germline BRCA1 and/or BRCA2 mutation (gBRCAm) substantially increases the risk of developing breast and/or ovarian cancer, as well as other tumor types such as prostate and pancreatic cancer [3-7].

Poly(ADP-ribose) polymerase (PARP) inhibitors block DNA damage repair in cells harboring a deficiency in homologous recombination repair (HRR), including mutations in BRCA1 and BRCA2 [8]. The PARP inhibitors olaparib and talazoparib have proven effective at targeting BRCA-mutated human epidermal growth factor receptor 2 (HER2)-negative metastatic breast cancers, including in the Phase 3 OlympiAD (NCT02000622) and EMBRACA (NCT01945775) trials [9-12]. The prevalence of a gBRCAm ranges from 1.2 to $8.8 \%$ in unselected breast cancer patient populations [13-19]. However, limited data exist on the prevalence of a gBRCAm in patients with HER2-negative metastatic breast cancer who require treatment with a first-line systemic chemotherapy regimen. Such data would provide an estimate of the size of the patient population who may be appropriate candidates to receive treatment with a PARP inhibitor for gBRCAm metastatic breast cancer.

Here, we present data from the global observational BREAKOUT study (NCT03078036) in patients with HER2-negative metastatic breast cancer being treated with first-line chemotherapy, the primary objective of which was to estimate the prevalence of gBRCAm in this patient population.

\section{Methods}

\section{Study design}

BREAKOUT was an observational, cross-sectional study, with a nested prospective cohort component (Fig. 1). The study design was based on the Phase $3 \mathrm{~b}$ real-world, open-label, single-arm LUCY study (NCT03286842) [20]. In the LUCY study, patients with a gBRCAm HER2-negative metastatic breast cancer were being treated with olaparib following no more than two prior lines of chemotherapy in the metastatic setting. BREAKOUT study sites were selected for their willingness to participate in the study and were requested to enroll sequential patients with HER2-negative metastatic breast cancer. See Additional file 1 for a list of participating study sites. The primary objective of BREAKOUT was to assess the prevalence of gBRCAm in a global population of patients with HER2-negative metastatic breast cancer who were not selected on the basis of risk factors for

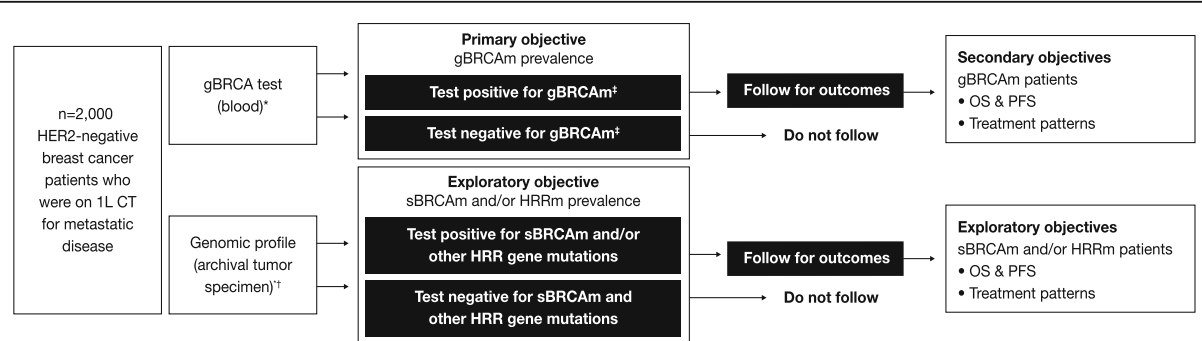

Fig. 1 BREAKOUT study design. BRCA, breast cancer susceptibility gene; $C T$, chemotherapy; gBRCAm, germline BRCA mutation; HER2, human epidermal growth factor receptor-2; HRRm, homologous recombination repair gene mutation; OS, overall survival; PFS, progression-free survival; sBRCAm, somatic BRCA mutation. *Blood/tumor testing occurred concurrently to the extent possible. ${ }^{\dagger}$ Foundation Medicine Inc. (Cambridge, Massachusetts, USA) Lynparza HRR assay was used to test for HRR gene mutations. "Positive: deleterious mutation; suspected deleterious. Negative: no deleterious mutation detected; no mutation detected; favor polymorphism; variant of uncertain significance; BRCA wildtype 
gBRCAm. For patients who tested positive for a gBRCAm, the planned secondary objectives included assessment of treatment patterns by line of therapy and prospective evaluation of clinical outcomes, including progression-free survival and overall survival.

In order to minimize selection bias, patients were selected regardless of demographic characteristics, presence of risk factors for gBRCAm breast cancer, or previously known gBRCAm status.

For patients who tested negative for a gBRCAm (gBRCAwt), archival tumor specimens were obtained where possible, to test for somatic BRCA mutations (sBRCAm) and other HRR gene mutations, using an investigational clinical trial assay, based on the FoundationOne ${ }^{\circ} \mathrm{CDx}$ platform (Foundation Medicine Inc., Cambridge, Massachusetts, USA). The HRR genes evaluated were $B R C A 1$, BRCA2, ATM, RAD51B, RAD51C, RAD51D, RAD54L, BRIP1, FANCL, PALB2, BARD1, CHEK1, CHEK2, CDK12, and $P P P 2 R 2 A$.

The planned sample size was 2000 patients with HER2negative metastatic breast cancer. Study enrollment was terminated early due to poor recruitment and, therefore, only patients' first-line cytotoxic chemotherapy regimens were collected for analysis. Data on subsequent therapies for metastatic breast cancer, progression-free survival, and overall survival were not collected due to the limited number of patients and absence of follow-up data.

Data including baseline patient demographic and disease characteristics, medical history, and treatment history were collected from individual patient records. Family history of breast and/or ovarian cancer in firstor second-degree relatives was also collected. Patients' gBRCAm status was determined by testing baseline blood samples (in a central laboratory, or in Japan using the Myriad BRACAnalysis CDx ${ }^{\circ}$ test) or, where available, using existing gBRCAm test results that had been obtained by local or central testing, as per local practice.

gBRCAm results were classified as positive (deleterious gBRCA1 and/or gBRCA2 mutation; genetic variant suspected deleterious), negative (gBRCAwt; genetic variant of uncertain significance; genetic variant, favor polymorphism; no mutation/deleterious mutation detected), or not determined.

\section{Patient population}

Eligible patients had started a first-line chemotherapy regimen for HER2-negative metastatic breast cancer within 90 days prior to enrollment. First-line chemotherapy was defined as the first chemotherapy given in the metastatic setting. Patients with hormone receptor (HR)positive metastatic breast cancer had developed disease progression on or after prior endocrine therapy, or were considered unsuitable for endocrine therapy. Patients who had received prior PARP inhibitor therapy were not eligible for the study.

\section{Statistical analysis}

The full analysis set (FAS) comprised all enrolled, eligible patients who had available gBRCAm test results. An exploratory subgroup included all patients in the FAS who had been tested for sBRCAm and/or HRR gene mutations, including those whose status could not be determined (e.g., due to the sample not being evaluable).

The prevalence of a gBRCAm was determined using the number of patients who were tested and had a valid result for gBRCAm status as the denominator and the number of patients who were gBRCAm-positive as the numerator. The prevalence of a gBRCAm according to demographic variables, disease characteristics, comorbidities, and choice of first-line therapy regimens was also evaluated.

Appropriate descriptive statistics were provided for continuous and categorical variables.

\section{Results}

Between March 2017 and April 2018, 384 patients were screened and consented to study enrollment; 43 patients were excluded from the analysis -4 for whom a blood sample for gBRCAm testing was not available and 39 for not meeting prespecified eligibility criteria-resulting in 341 patients with HER2-negative metastatic breast cancer comprising the FAS (Fig. 2).

Patients were enrolled across 14 countries: Australia $(n=5)$, Bulgaria $(n=15)$, Canada $(n=8)$, Hungary $(n=3)$, Italy $(n=7)$, Japan $(n=44)$, Poland $(n=13)$, Russia $(n=32)$, South Korea $(n=45)$, Spain $(n=18)$, Taiwan $(n=15)$, Turkey $(n=79)$, the UK $(n=32)$, and the USA $(n=25)$.

\section{gBRCAm prevalence}

Of the 341 patients included in the FAS, 33 (9.7\%; 95\% confidence interval [CI] 6.8\%, 13.3\%) harbored a gBRCAm (Table 1). Mutations were detected in gBRCA1 alone in 16 patients (4.7\%; 95\% CI 2.7\%, 7.5\%), in gBRCA2 alone in 12 patients (3.5\%; 95\% CI 1.8\%, 6.1\%), and in both gBRCA1 and $\mathrm{gBRCA} 2$ in 5 patients $(1.5 \%$; $95 \%$ CI $0.5 \%, 3.4 \%)$. A total of 30 patients underwent gBRCAm testing prior to baseline; 8 (26.7\%) of these patients had a gBRCAm.

Subgroup analyses showed the prevalence of gBRCAm was $9.0 \%$ in European patients $(n=18 / 199 ; 95 \%$ CI $5.4 \%$, 13.9\%), 9.1\% in North American patients $(n=3 / 33$; $95 \%$ CI $1.9 \%, 24.3 \%$ ), and $10.6 \%$ in Asian patients (Japan, South Korea, and Taiwan; $n=11 / 104 ; 95 \%$ CI $5.4 \%$, 18.1\%) (Table 1).

\section{SBRCA and HRR gene mutations}

In total, 64 patients with gBRCAwt who had archival breast cancer tissue were tested for HRR gene mutations and sBRCAm. The prevalence of sBRCAm was 


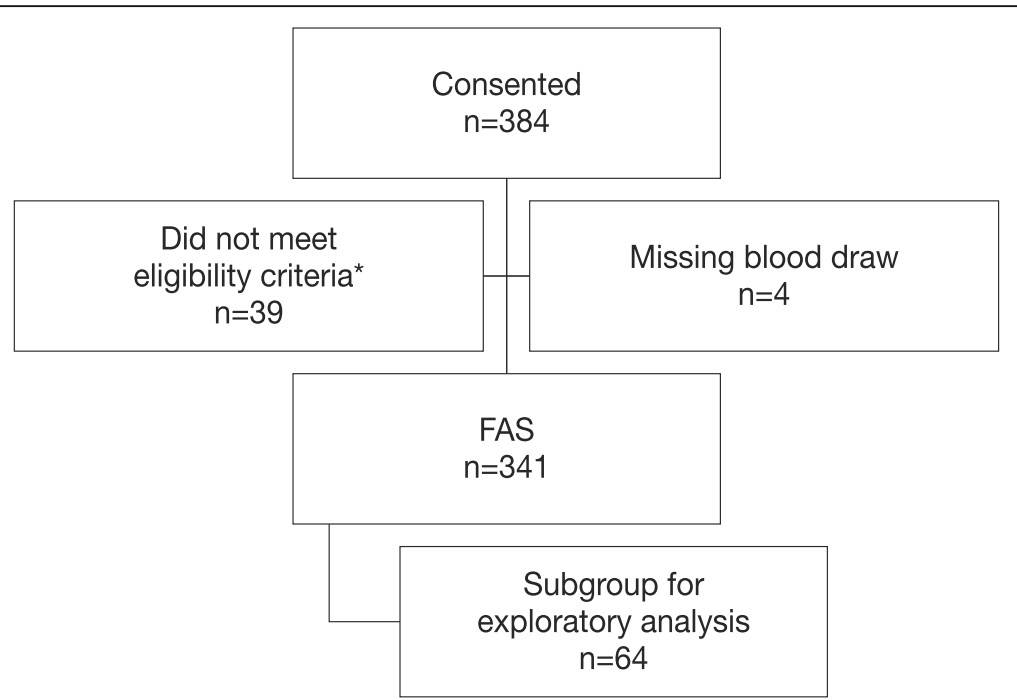

Fig. 2 Patient disposition. FAS, full analysis set. *39 patients did not meet eligibility criteria; 29 patients had not initiated treatment with first-line systemic cytotoxic chemotherapy for metastatic breast cancer in the past 90 days and, at that time, were considered to have exhausted endocrine therapy options if hormone receptor-positive; 9 patients had no evidence of metastatic disease; and 1 patient consented after the termination of the study

$6.3 \%(n=4 / 64 ; 95 \%$ CI $1.7 \%, 15.2 \%)$. One patient had sBRCA1 mutation only $(1.6 \%$; $95 \%$ CI $0.0 \%, 8.4 \%)$ and three patients had sBRCA2 mutation only $(4.7 \%$; $95 \%$ CI $1.0 \%, 13.1 \%$ ) (Fig. 3). The incidence of sBRCAm in patients with an existing gBRCAm was not assessed in the BREAKOUT study. The prevalence of any HRR gene mutation overall was $14.1 \%(n=9 / 64 ; 95 \%$ CI $6.6 \%$, $25.0 \%)$, including mutations in ATM $(n=2), B R C A 1$ $(n=1), \operatorname{BRCA2}(n=3), \operatorname{CHEK} 2(n=1), \operatorname{PALB2}(n=1)$, and $R A D 51 B(n=1)$ (Fig. 3).

\section{Patient demographic and clinical characteristics}

Baseline demographic and clinical characteristics for patients in the FAS are shown in Table 2. Median (range) age at enrollment was 56 (25-89) years. Median (range) age at initial breast cancer diagnosis was 40 (24-71) years in patients with a gBRCAm and 52 (24-86) years in patients with gBRCAwt.

Most patients were postmenopausal at enrollment ( $n=256 / 340 ; 75.3 \%)$. The proportion of postmenopausal patients was lower in the gBRCAm group (57.6\%) compared with the gBRCAwt group (77.2\%). In the FAS,
$58.1 \%$ of patients $(n=198 / 341)$ were postmenopausal at initial breast cancer diagnosis. Most patients with gBRCAwt were postmenopausal at initial breast cancer diagnosis $(n=186 / 308 ; 60.4 \%)$, compared with $36.4 \%$ of patients with a gBRCAm $(n=12 / 33)$.

Median time from initial breast cancer diagnosis to enrollment was 28.1 months (interquartile range 13.4-73.0) in patients with a gBRCAm and was similar at 29.9 months (interquartile range 6.3-78.2) in patients with gBRCAwt (Table 2).

Disease stage at initial breast cancer diagnosis in the FAS was stage 0 in $2.7 \%(n=9 / 336)$ of patients, stage I in $9.2 \%(31 / 336)$ of patients, stage II in $34.8 \%(n=117 / 336)$ of patients, stage III in $26.2 \%$ ( $n=88 / 336)$ of patients, and stage IV in $27.1 \%$ (91/336) of patients, with similar distribution among patients with a gBRCAm (Table 2). With respect to nodal status at initial breast cancer diagnosis, in the FAS, $25.3 \%$ of patients had node stage N0 (negative) and $33.5 \%$ had node stage N1 disease. Tumor grade at initial breast cancer diagnosis was poorly differentiated in $35.1 \%$ of patients $(n=118 / 336)$ and moderately differentiated in $33.0 \%$ of patients $(n=111 / 336)$ (Table 2). In

Table 1 gBRCAm prevalence by region of enrollment (FAS)

\begin{tabular}{llllll}
\hline & Asia $(\boldsymbol{N}=\mathbf{1 0 4})$ & Europe $(\boldsymbol{N}=\mathbf{1 9 9})$ & North America $(\boldsymbol{N}=\mathbf{3 3})$ & Australia/Oceania $(\boldsymbol{N}=\mathbf{5})$ & FAS $(\boldsymbol{N}=\mathbf{3 4 1})$ \\
\hline $\begin{array}{l}\text { Positive for a gBRCAm, } \\
n(\%[95 \% \text { CI]) }\end{array}$ & $11(10.6[5.4,18.1])$ & $18(9.0[5.4,13.9])$ & $3(9.1[1.9,24.3])$ & $1(20.0[0.5,71.6])$ & $33(9.7[6.8,13.3])$ \\
gBRCA1m only, $n(\%[95 \% \mathrm{CI}])$ & $6(5.8[2.1,12.1])$ & $10(5.0[2.4,9.0])$ & $0(0.0[0.0,10.6])$ & $0(0.0[0.0,52.2])$ & $16(4.7[2.7,7.5])$ \\
gBRCA2m only, $n(\%[95 \% \mathrm{CI}])$ & $5(4.8[1.6,10.9])$ & $6(3.0[1.1,6.4])$ & $1(3.0[0.1,15.8])$ & $0(0.0[0.0,52.2])$ & $12(3.5[1.8,6.1])$ \\
$\begin{array}{l}\text { Both gBRCA1m and gBRCA2m, } \\
n(\%[95 \% \text { CI]) }\end{array}$ & $0(0.0[0.0,3.5])$ & $2(1.0[0.1,3.6])$ & $2(6.1[0.7,20.2])$ & $1(20.0[0.5,71.6])$ & $5(1.5[0.5,3.4])$
\end{tabular}




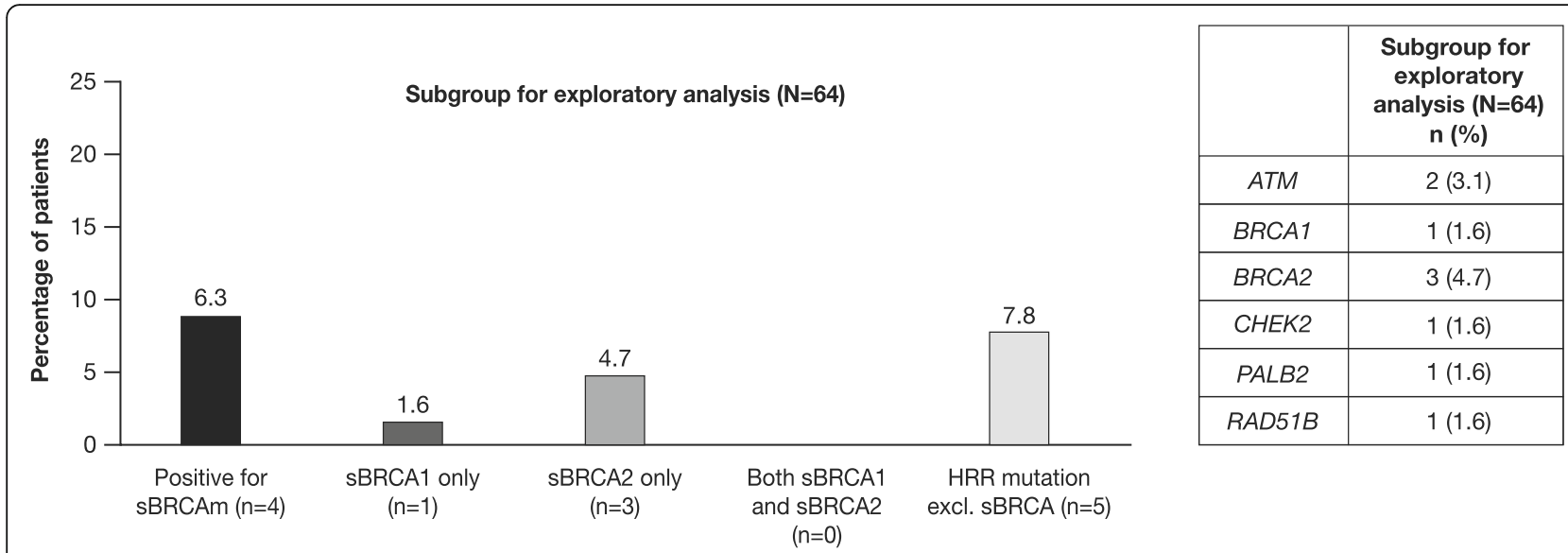

Fig. 3 Prevalence of sBRCAm and other HRR gene mutations (subgroup for exploratory analysis). BRCA1, breast cancer susceptibility gene 1; $B R C A 2$, breast cancer susceptibility gene 2; HRRm, homologous recombination repair gene mutation; sBRCAm, somatic BRCA mutation; sBRCA1, somatic BRCA1 mutation; SBRCA2, somatic BRCA2 mutation

comparison with all patients in the FAS, more patients with a gBRCAm had poorly differentiated cancers $(45.5 \%$; $n=15 / 33)$ and $30.3 \% \quad(n=10 / 33)$ of patients with a gBRCAm had moderately differentiated tumors.

Overall, $52.0 \% \quad(n=156 / 300)$ of patients with gBRCAwt had received chemotherapy prior to metastatic disease, compared with $64.5 \% \quad(n=20 / 31)$ of patients with a gBRCAm, and 39.0\% $(n=119 / 305)$ of patients with gBRCAwt received non-chemotherapy treatments prior to metastatic disease, compared with $30.3 \%(n=10 / 33)$ of patients with a gBRCAm. Nonchemotherapy treatments included endocrine therapy (including tamoxifen, letrozole, anastrozole, exemestane, and fulvestrant), everolimus $(n=1)$, and bevacizumab $(n=1)$ (Table 2) and were mostly (77.9\%) used as adjuvant therapy.

Non-chemotherapy treatments (including letrozole, fulvestrant, and exemestane) were administered for metastatic disease prior to receiving first-line chemotherapy in $24.2 \%(n=8 / 33)$ of patients with a gBRCAm and $30.9 \%(n=95 / 307)$ of patients with gBRCAwt.

Similar proportions of patients with a gBRCAm or gBRCAwt had HR-positive (estrogen receptor- and/or progesterone receptor-positive) versus HR-negative HER2negative metastatic breast cancer with gBRCAwt (Table 2).

A family history of breast and/or ovarian cancer in a first- or second-degree relative was recorded for $45.5 \%$ ( $n=15 / 33)$ of patients with a gBRCAm, compared with $16.6 \%(n=51 / 307)$ of patients with gBRCAwt (Table 2$)$.

\section{Subgroup analyses by risk factors for gBRCAm Age at initial breast cancer diagnosis}

The prevalence of a gBRCAm was $12.9 \%$ ( $n=22 / 171$; $95 \%$ CI $8.2 \%, 18.8 \%)$ in patients aged $\leq 50$ years at initial breast cancer diagnosis and was lower at $5.4 \%$ $(n=9 / 167 ; 95 \%$ CI $2.5 \%, 10.0 \%)$ in patients $>50$ years of age at initial breast cancer diagnosis (Table 3). The prevalence of a $g B R C A 1$ mutation was higher in patients aged $\leq 50$ years at initial breast cancer diagnosis $(7.6 \%$; $n=13 / 171 ; 95 \%$ CI $4.1 \%, 12.6 \%)$ than in patients aged $>50$ years at initial breast cancer diagnosis $(1.2 \% ; n=2 / 167$; $95 \%$ CI $0.1 \%, 4.3 \%)$, whereas prevalence of a gBRCA2 mutation was similar in patients aged $\leq 50$ years and $>50$ years, at $3.5 \%$ (95\% CI 1.3\%, 7.5\%) and 3.0\% (95\% CI 1.0\%, 6.8\%), respectively.

\section{Hormone receptor status}

The overall prevalence of a gBRCAm was similar with respect to HR status: $9.3 \%(n=20 / 215 ; 95 \%$ CI $5.8 \%$, $14.0 \%)$ in patients with HR-positive disease (6 patients in gBRCA1 only, 10 in gBRCA2 only, and 4 in both gBRCA1 and gBRCA2) and 9.2\% $(n=11 / 119 ; 95 \% \mathrm{CI}$ $4.7 \%, 15.9 \%)$ in patients with $\mathrm{HR}$-negative disease (9 patients in gBRCA1 only and 2 in gBRCA2 only) (Table 3). However, the prevalence of a $\mathrm{gBRCA1} \mathrm{mu}-$ tation was $7.6 \%(95 \%$ CI 3.5\%, 13.9\%) in patients with HR-negative metastatic breast cancer and 2.8\% (95\% CI $1.0 \%, 6.0 \%)$ in patients with HR-positive metastatic breast cancer, while a gBRCA2 mutation was more frequent in those with HR-positive metastatic breast cancer, compared with HR-negative metastatic breast cancer $(4.7 \%$ [95\% CI 2.3\%, 8.4\%] and $1.7 \%$ [95\% CI $0.2 \%, 5.9 \%]$, respectively).

\section{Family history of breast and/or ovarian cancer}

The prevalence of a gBRCAm was higher (22.7\%; $n=15 / 66 ; 95 \%$ CI $13.3 \%, 34.7 \%)$ in the subgroup of patients with a family history of breast and/or ovarian cancer, compared with patients without a family history of breast and/or ovarian cancer $(6.6 \% ; n=18 / 274 ; 95 \%$ 
Table 2 Baseline patient demographic and disease characteristics (FAS)

\begin{tabular}{|c|c|c|c|}
\hline & \multicolumn{2}{|l|}{ gBRCAm status } & \multirow[b]{2}{*}{ FAS $(N=341)$} \\
\hline & Positive $(N=33)$ & Negative $(N=308)$ & \\
\hline \multicolumn{4}{|l|}{ Age at enrollment (years) } \\
\hline$n$ & 33 & 308 & 341 \\
\hline Median (range) & $47.0(25-71)$ & $56.5(29-89)$ & $56.0(25-89)$ \\
\hline \multicolumn{4}{|l|}{ Race, $n(\%)$} \\
\hline$n$ & 28 & 267 & 295 \\
\hline White & $22(78.6)$ & $201(75.3)$ & $223(75.6)$ \\
\hline Black or African American & 0 & $4(1.5)$ & $4(1.4)$ \\
\hline Asian & $6(21.4)$ & $60(22.5)$ & $66(22.4)$ \\
\hline Native Hawaiian or Other Pacific Islander & 0 & $1(0.4)$ & $1(0.3)$ \\
\hline Other & 0 & $1(0.4)$ & $1(0.3)$ \\
\hline \multicolumn{4}{|l|}{ Age at initial breast cancer diagnosis (years) } \\
\hline$n$ & 31 & 307 & 338 \\
\hline Median (range) & $40.0(24-71)$ & $52.0(24-86)$ & $50.0(24-86)$ \\
\hline \multicolumn{4}{|c|}{ Family history of breast and/or ovarian cancer, $n(\%)$} \\
\hline$n$ & 33 & 307 & 340 \\
\hline Yes & $15(45.5)$ & $51(16.6)$ & $66(19.4)$ \\
\hline No & $18(54.5)$ & $256(83.4)$ & $274(80.6)$ \\
\hline \multicolumn{4}{|c|}{ Time since initial breast cancer diagnosis to enrollment (months) } \\
\hline$n$ & 31 & 307 & 338 \\
\hline Median (interquartile range) & $28.1(13.4-73.0)$ & $29.9(6.3-78.2)$ & $29.8(7.2-76.8)$ \\
\hline \multicolumn{4}{|l|}{ Sites of metastatic disease, $n(\%)$} \\
\hline$n$ & 33 & 308 & 341 \\
\hline Bone and locomotor & $13(39.4)$ & $162(52.6)$ & $175(51.3)$ \\
\hline Lymph nodes & $15(45.5)$ & $134(43.5)$ & $149(43.7)$ \\
\hline Respiratory & $10(30.3)$ & $86(27.9)$ & $96(28.2)$ \\
\hline Liver & $8(24.2)$ & $69(22.4)$ & $77(22.6)$ \\
\hline Other metastatic sites & $23(69.7)$ & $194(63.0)$ & $217(63.6)$ \\
\hline \multicolumn{4}{|l|}{ HR status at most recent assessment, $n(\%)$} \\
\hline$n$ & 31 & 303 & 334 \\
\hline Positive & $20(64.5)$ & $195(64.4)$ & $215(64.4)$ \\
\hline \multicolumn{4}{|c|}{ AJCC stage at initial breast cancer diagnosis, $n(\%)$} \\
\hline$n$ & 33 & 303 & 336 \\
\hline 0 & $1(3.0)$ & $8(2.6)$ & $9(2.7)$ \\
\hline Stage I (I, A, B, C) & $3(9.1)$ & $28(9.2)$ & $31(9.2)$ \\
\hline Stage $\|(\|, A, B, C)$ & $13(39.4)$ & $104(34.3)$ & $117(34.8)$ \\
\hline Stage III (III, A, B, C) & $8(24.2)$ & $80(26.4)$ & $88(26.2)$ \\
\hline Stage IV (IV, A, B, C) & $8(24.2)$ & $83(27.4)$ & $91(27.1)$ \\
\hline \multicolumn{4}{|l|}{ Nodal status at original diagnosis, $n(\%)$} \\
\hline$n$ & 33 & 307 & 340 \\
\hline No & $12(36.4)$ & $74(24.1)$ & $86(25.3)$ \\
\hline N1 & $8(24.2)$ & $106(34.5)$ & $114(33.5)$ \\
\hline N2 & $7(21.2)$ & $54(17.5)$ & $61(17.9)$ \\
\hline N3 & $5(15.2)$ & $38(12.4)$ & $43(12.6)$ \\
\hline
\end{tabular}


Table 2 Baseline patient demographic and disease characteristics (FAS) (Continued)

\begin{tabular}{|c|c|c|c|}
\hline & \multicolumn{2}{|l|}{ gBRCAm status } & \multirow[b]{2}{*}{ FAS $(N=341)$} \\
\hline & Positive $(N=33)$ & Negative $(N=308)$ & \\
\hline pNO & 0 & $4(1.3)$ & $4(1.2)$ \\
\hline NX & $1(3.0)$ & $29(9.4)$ & $30(8.8)$ \\
\hline $\mathrm{N} 1 \mathrm{a}$ & 0 & $2(0.7)$ & $2(0.6)$ \\
\hline \multicolumn{4}{|c|}{ Tumor grade at original diagnosis, $n(\%)$} \\
\hline$n$ & 33 & 303 & 336 \\
\hline X (undetermined) & $3(9.1)$ & $65(21.5)$ & $68(20.2)$ \\
\hline 1 (well differentiated) & $2(6.1)$ & $22(7.3)$ & $24(7.1)$ \\
\hline 2 (moderately differentiated) & $10(30.3)$ & $101(33.3)$ & $111(33.0)$ \\
\hline 3 (poorly differentiated) & $15(45.5)$ & $103(34.0)$ & $118(35.1)$ \\
\hline 4 (undifferentiated) & 0 & $3(1.0)$ & $3(0.9)$ \\
\hline High grade* & $3(9.1)$ & $9(3.0)$ & $12(3.6)$ \\
\hline \multicolumn{4}{|c|}{ Non-chemotherapy treatment prior to metastatic disease, $n(\%)^{\dagger}$} \\
\hline$n$ & 33 & 305 & 338 \\
\hline Tamoxifen & $7(21.2)$ & $74(24.3)$ & $81(24.0)$ \\
\hline Letrozole & $3(9.1)$ & $37(12.1)$ & $40(11.8)$ \\
\hline Anastrozole & $2(6.1)$ & $35(11.5)$ & $37(10.9)$ \\
\hline Exemestane & $1(3.0)$ & $3(1.0)$ & $4(1.2)$ \\
\hline Fulvestrant & 0 & $8(2.6)$ & $8(2.4)$ \\
\hline Everolimus & $1(3.0)$ & 0 & $1(0.3)$ \\
\hline Other $^{\ddagger}$ & $1(3.0)$ & $11(3.6)$ & $12(3.6)$ \\
\hline
\end{tabular}

AJCC American Joint Committee on Cancer, BRCA breast cancer susceptibility gene, eCRF electronic case report form, FAS full analysis set, $g B R C A$ germline BRCA mutation, HR hormone receptor, $S D$ standard deviation

*High grade was listed as an additional category in the eCRF and is based on the Nottingham grading system (total score: 8-9)

${ }^{\dagger} \mathrm{A}$ patient may have had more than one type of non-chemotherapy treatment

${ }^{\ddagger}$ Additional non-chemotherapy treatments to those listed in the table included leuprorelin/leuprorelin acetate $(n=5)$, toremifene/toremifene citrate $(n=3)$,

bevacizumab $(n=1)$, goserelin $(n=1)$, tamoxifen citrate $(n=1)$, and trastuzumab $(n=1)$

CI $3.9 \%, 10.2 \%$ ) (Table 3). In patients with a family history of breast and/or ovarian cancer, mutations in gBRCA1 (12.1\% [95\% CI 5.4\%, 22.5\%]) and gBRCA2 (9.1\% [95\% CI 3.4\%, 18.7\%]) were more prevalent than in patients without a family history of breast and/or ovarian cancer $(2.9 \%$ [95\% CI 1.3\%, 5.7\%] and 2.2\% [95\% CI $0.8 \%, 4.7 \%]$, respectively).
Presence of $\geq 1$ risk factor for having a gBRCAm

gBRCAm prevalence was $10.4 \%(n=26 / 250$; $95 \%$ CI $6.9 \%$, $14.9 \%$ ) in patients who had at least one risk factor for having a gBRCAm (family history of breast and/or ovarian cancer; age at initial breast cancer diagnosis $\leq 50$ years; or triplenegative breast cancer), compared with 5.8\% ( $n=5 / 86$; 95\% CI 1.9\%, 13.0\%) in patients without any of these risk factors.

Table 3 gBRCAm prevalence by risk factors for gBRCAm (FAS)

\begin{tabular}{|c|c|c|c|c|}
\hline Risk factor & $\begin{array}{l}\text { Positive for a gBRCAm, } \\
n(\%[95 \% \mathrm{CI}])\end{array}$ & $\begin{array}{l}\text { gBRCA1m only, } \\
n(\%[95 \% \mathrm{Cl}])\end{array}$ & $\begin{array}{l}\text { gBRCA2m only, } \\
n(\%[95 \% \mathrm{Cl}])\end{array}$ & 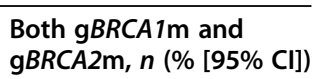 \\
\hline \multicolumn{5}{|c|}{ Age at initial breast cancer diagnosis } \\
\hline$\leq 50$ years $(N=171)$ & $22(12.9[8.2,18.8])$ & $13(7.6[4.1,12.6])$ & $6(3.5[1.3,7.5])$ & $3(1.8[0.4,5.0])$ \\
\hline$>50$ years $(N=167)$ & $9(5.4[2.5,10.0])$ & $2(1.2[0.1,4.3])$ & $5(3.0[1.0,6.8])$ & $2(1.2[0.1,4.3])$ \\
\hline \multicolumn{5}{|l|}{ HR status } \\
\hline HR-positive $(N=215)$ & $20(9.3[5.8,14.0])$ & $6(2.8[1.0,6.0])$ & $10(4.7[2.3,8.4])$ & $4(1.9[0.5,4.7])$ \\
\hline HR-negative ( $\mathrm{N}=119)$ & $11(9.2[4.7,15.9])$ & $9(7.6[3.5,13.9])$ & $2(1.7[0.2,5.9])$ & $0(0.0[0.0,3.1])$ \\
\hline \multicolumn{5}{|c|}{ Family history of breast and/or ovarian cancer } \\
\hline Yes $(N=66)$ & $15(22.7[13.3,34.7])$ & $8(12.1[5.4,22.5])$ & $6(9.1[3.4,18.7])$ & $1(1.5[0.0,8.2])$ \\
\hline No $(N=274)$ & $18(6.6[3.9,10.2])$ & $8(2.9[1.3,5.7])$ & $6(2.2[0.8,4.7])$ & $4(1.5[0.4,3.7])$ \\
\hline
\end{tabular}

$B R C A$ breast cancer susceptibility gene, $C l$ confidence interval, $F A S$ full analysis set, $g B R C A m$ germline $B R C A$ mutation, $H R$ hormone receptor 


\section{First-line chemotherapy use}

Overall, most patients in the FAS received single-agent chemotherapy as their first-line chemotherapy regimen ( $n=196 / 341 ; 57.5 \%)$ (Table 4$)$, while $54.5 \%(n=18 / 33)$ of patients with a gBRCAm received combination chemotherapy with $\geq 2$ agents as their first-line chemotherapy regimen. Overall, the most frequently used single-agent regimens $(>10 \%)$ were paclitaxel $(n=75 / 196 ; 38.3 \%)$, capecitabine $(n=42 / 196 ; 21.4 \%)$, and docetaxel $(n=24 /$ $196 ; 12.2 \%)$, while the most frequently used combination regimen was paclitaxel/bevacizumab $(n=21 / 145 ; 14.5 \%)$ (see Additional file 2).

The most frequently used cytotoxic chemotherapy agent as first-line therapy, either as a single agent or in combination, regardless of gBRCAm status, was paclitaxel $(n=$ $127 / 341 ; 37.2 \%)$, followed by cyclophosphamide $(n=60$ / $341 ; 17.6 \%)$, capecitabine $(n=57 / 341 ; 16.7 \%)$, docetaxel ( $n=48 / 341 ; 14.1 \%)$, carboplatin $(n=31 / 341 ; 9.1 \%)$, doxorubicin $(n=29 / 341 ; 8.5 \%)$, and gemcitabine $(n=28 / 341$; $8.2 \%$ ) (Table 4). First-line cytotoxic chemotherapy regimens are detailed in Additional file 2.

\section{Discussion}

The objectives of the BREAKOUT study were to estimate the true prevalence of gBRCAm in patients with HER2-negative metastatic breast cancer, by minimizing selection bias, and to generate observational outcome data. Outcome data, however, were not obtained from the BREAKOUT study, as the study was terminated early due to inadequate recruitment.

The estimate of gBRCAm prevalence in the observational BREAKOUT study was 9.7\% (95\% CI 6.8\%, 13.3\%) among a cohort of 341 patients with HER2-negative metastatic breast cancer being treated with first-line cytotoxic chemotherapy. Previous studies in unselected patient populations reported the prevalence of a $\mathrm{g} B R C A 1 \mathrm{~m}$ and a gBRCA2m to be between 1.2 and $8.8 \%$ [13-19]. In contrast, in a US study of 119 patients with HER2-negative metastatic breast cancer who were referred for mutation testing, and who likely had perceived risk factors for having a gBRCAm, the estimated prevalence was higher than that observed in the BREAKOUT population, at $24.4 \%$ [21]. Overall there were no notable differences in the prevalence of a gBRCAm with regards to HR status, sites of metastases, time from initial breast cancer diagnosis to entry into the BREAKOUT study, and physicians' choice of first-line cytotoxic chemotherapy regimens.

In patients with at least one risk factor (family history of breast and/or ovarian cancer; age at initial breast cancer diagnosis $\leq 50$ years; or triple-negative breast cancer), gBRCAm prevalence was $10.4 \%$ in the BREAKOUT

Table 4 Cytotoxic chemotherapies administered as first-line therapy for metastatic breast cancer (FAS)

\begin{tabular}{|c|c|c|c|}
\hline & \multicolumn{2}{|l|}{ gBRCAm status } & \multirow[b]{2}{*}{ FAS $(N=341$} \\
\hline & Positive $(N=33)$ & Negative $(N=308)$ & \\
\hline \multicolumn{4}{|c|}{ Number of unique agents received as first-line therapy, $n(\%)^{*}$} \\
\hline 1 & $15(45.5)$ & $181(58.8)$ & $196(57.5)$ \\
\hline 2 & $15(45.5)$ & $105(34.1)$ & $120(35.2)$ \\
\hline 3 & $1(3.0)$ & $16(5.2)$ & $17(5.0)$ \\
\hline $4+$ & $2(6.1)$ & $6(1.9)$ & $8(2.3)$ \\
\hline \multicolumn{4}{|c|}{ Cytotoxic chemotherapy agent (in $>5 \%$ of patients), $n(\%)^{*}$} \\
\hline Paclitaxel & $12(36.4)$ & $115(37.3)$ & $127(37.2)$ \\
\hline Cyclophosphamide & $5(15.2)$ & $55(17.9)$ & $60(17.6)$ \\
\hline Capecitabine & $7(21.2)$ & $50(16.2)$ & $57(16.7)$ \\
\hline Docetaxel & $6(18.2)$ & $42(13.6)$ & $48(14.1)$ \\
\hline Carboplatin & $5(15.2)$ & $26(8.4)$ & $31(9.1)$ \\
\hline Doxorubicin & $3(9.1)$ & $26(8.4)$ & $29(8.5)$ \\
\hline Gemcitabine & $4(12.1)$ & $24(7.8)$ & $28(8.2)$ \\
\hline Bevacizumab & $4(12.1)$ & $22(7.1)$ & $26(7.6)$ \\
\hline Cisplatin & $1(3.0)$ & $22(7.1)$ & $23(6.7)$ \\
\hline Epirubicin & $2(6.1)$ & $19(6.2)$ & $21(6.2)$ \\
\hline
\end{tabular}

First-line cytotoxic chemotherapy was defined as the first chemotherapy given in the metastatic setting up to disease progression. The first-line chemotherapy start date should have occurred by the time of the latest date of metastatic diagnosis being made ( 30 days) and informed consent being given ( 90 days). The window to metastatic diagnosis date was defined to capture treatments given after the initial clinical/radiologic metastatic diagnosis $B R C A$ breast cancer susceptibility gene, FAS full analysis set, $g B R C A m$ germline $B R C A$ mutation

*If an agent was reported in two or more different regimens or treatment combinations in the first line, the agent was counted only once for that patient 
study, compared with $5.8 \%$ in patients without any of these risk factors. Also of note is that approximately half of the patients with a gBRCAm in BREAKOUT (54.5\%) did not have a family history of breast and/or ovarian cancer. This is notable as, although the number of patients involved is small, $5.8 \%$ is a sizeable proportion of patients whose gBRCAm could be missed if the criteria for gBRCAm testing in patients with HER2-negative metastatic breast cancer are based on the presence of risk factors, including family history of breast and/or ovarian cancer, as well as other tumor types such as prostate and pancreatic cancer [22]. Similar findings were observed in a US study in a population of patients with any-stage triple-negative breast cancer, in which $16 \%$ of patients with a gBRCAm did not have an established reason for gBRCAm testing and 10\% had limited family history knowledge of breast and/or ovarian cancer at any age [23]. The data from the BREAKOUT study support the National Comprehensive Cancer Network recommendation that all patients with HER2-negative metastatic breast cancer undergo gBRCAm testing [24].

The prevalence of sBRCAm in 64 gBRCAwt patients with archival tumor tissue was $6.3 \%$ (one patient with $\mathrm{s} B R C A 1$ and three with $\mathrm{s} B R C A 2)$. The prevalence of any HRR gene mutation (excluding $\mathrm{s} B R C A$ ) in this group was $7.8 \%$. There are very limited data that describe the prevalence of somatic or germline non-BRCA HRR gene mutations in patients with metastatic breast cancer. Data from the Cancer Genome Atlas Program (TCGA) show that $\sim 20 \%$ of basal-like breast cancers have a germline and/or somatic $B R C A 1$ or $B R C A 2$ variant [25]. In the ongoing PRAEGNANT (Prospective Academic Translational Research Network for the Optimization of Oncological Health Care Quality in the Adjuvant and Advanced Therapeutic Setting) registry study in Germany (NCT02338167), of 1462 patients with metastatic breast cancer receiving any therapeutic regimen who had available germline DNA from time of study entry and successful genotyping, $4.3 \%$ had a non-BRCA germline HRR gene mutation [26]. Results from the PRAEGNANT study are expected to provide further insights into the prevalence of germline HRR gene mutations and their impact on outcome [27]. In addition, the European AURORA study that is recruiting 1300 patients with metastatic breast cancer will likely generate data on the prevalence of germline and somatic HRR gene mutations and their impact on treatment outcomes [28].

This study had some limitations that could impact the generalizability of the results. With the gBRCAm testing costs covered by this observational study, investigators may have been more likely to enroll patients who were at higher risk for having a gBRCAm. However, the patient characteristics of the BREAKOUT metastatic breast cancer population, including age, menopausal status, and HR status, were similar to those described in other HER2-negative metastatic breast cancer patient populations that were unselected for gBRCAm status [29-33]. An additional limitation was that the BREAKOUT study excluded patients previously treated with PARP inhibitors and, therefore, participating study sites were more likely to be those in which patients had not been exposed to PARP inhibitors in clinical trials. This may have had some influence on the patient population, as well as the size and type of participating centers, and this might have contributed to the inadequate recruitment.

The smaller than planned sample size, due to the early termination of the study, had an impact on the level of precision and generalizability of the findings. The study was designed to describe the prevalence of a gBRCAm with a precision of approximately $\pm 2 \%$ and, with only 341 patients in the FAS, a precision of $6.5 \%$ was achieved.

The BREAKOUT study incorporated the findings of gBRCAm tests performed prior to the study if they were available. To avoid distortion by a possible overrepresentation of patients with a prior test, participating sites were instructed to enroll patients in consecutive order, regardless of the availability of prior $B R C A$ mutation test results. In the FAS, $8.8 \%(n=30)$ of patients were tested for gBRCAm status prior to the baseline, and of these, 8 patients $(26.7 \%)$ had a gBRCAm (these patients were repeat tested at baseline).

\section{Conclusions}

The global BREAKOUT study demonstrated that 9.7\% of patients with HER2-negative metastatic breast cancer who were receiving a first-line cytotoxic chemotherapy regimen had a gBRCAm and that $5.8 \%$ of the enrolled patients who had no standard risk factors had a gBRCAm.

The results help characterize the patient population who may benefit from PARP inhibitor therapy and highlight the need for broad gBRCAm testing of patients with HER2-negative metastatic breast cancer.

\section{Supplementary information}

Supplementary information accompanies this paper at https://doi.org/10. 1186/s13058-020-01349-9.

Additional file 1: Table S1. List of participating sites. This table details the sites that participated in the BREAKOUT study.

Additional file 2: Table S2. First-line cytotoxic chemotherapy regimens in $>5 \%$ of patients (FAS). This table details first-line cytotoxic therapy regimens (single agent and combination agent).

Abbreviations

BC: Breast cancer; BRCA: Breast cancer susceptibility gene; Cl: Confidence interval; CT: Chemotherapy; FAS: Full analysis set; gBRCAm: Germline BRCA mutation; gBRCAwt: Germline BRCA wildtype; HER2: Human epidermal 
growth factor receptor 2; HR: Hormone receptor; HRR: Homologous recombination repair; HRRm: Homologous recombination repair gene mutation; MBC: Metastatic breast cancer; OS: Overall survival; PARP: Poly(ADP-ribose) polymerase; PFS: Progression-free survival; PRAEGNAN T: Prospective Academic Translational Research Network for the Optimization of Oncological Health Care Quality in the Adjuvant and Advanced Therapeutic Setting; sBRCAm: Somatic BRCA mutation; sHRRm: Somatic homologous recombination repair gene mutation; SD: Standard deviation

\section{Acknowledgements}

The authors wish to thank the patients, their families/caregivers, investigators, and study personnel.

Medical writing support, under the direction of the authors, was provided by Laura Fullerton-Batten, PhD, CMC Connect, McCann Health Medical Communications, funded by AstraZeneca, Cambridge, UK, in accordance with Good Publication Practice (GPP3) guidelines (Ann Intern Med. 2015;163(6):461-464).

\section{Authors' contributions}

JO'S, CB-M, MC, and SO carried out data collection, analysis, and interpretation. TD, GW, and JB carried out data analysis and interpretation. All authors drafted and revised the manuscript and approved the final draft.

\section{Funding}

This study was sponsored by AstraZeneca and is part of an alliance between AstraZeneca and Merck Sharp \& Dohme Corp., a subsidiary of Merck \& Co., Inc., Kenilworth, NJ, USA (MSD).

\section{Availability of data and materials}

Data underlying the findings described in this manuscript may be obtained in accordance with AstraZeneca's data-sharing policy, described at https:// astrazenecagrouptrials.pharmacm.com/ST/Submission/Disclosure

\section{Ethics approval and consent to participate}

An independent ethics committee or institutional review board approved the final protocol at each study site. All patients provided written informed consent.

\section{Consent for publication}

Not applicable.

\section{Competing interests}

Dr. Joyce O'Shaughnessy has received honoraria for consulting and advisory boards from AbbVie, Agendia, Amgen, AstraZeneca, Bristol-Myers Squibb, Celgene Corporation, Eisai, Eli Lilly, Genentech, Genomic Health, GRAIL, Heron Therapeutics, Immunomedics, Ipsen Biopharmaceuticals, Jounce Therapeutics, Merck, Myriad, Novartis, Odonate Therapeutics, Pfizer, Puma Biotechnology, Roche, Seattle Genetics, and Syndax Pharmaceuticals.

Dr. Christine Brezden-Masley has received honoraria for consulting and advisory boards from Amgen, AstraZeneca, Eli Lilly, Genomic Health, Merck, Myriad, Novartis, Pfizer, Roche, and Taiho. She has received travel grants from Amgen, AstraZeneca, and Roche.

Dr. Marina Cazzaniga reports no competing interests.

Dr. Tapashi Dalvi, Dr. Graham Walker, and Dr. James Bennett are employees or contracted employees of AstraZeneca and may own stock.

Dr. Shozo Ohsumi has received honoraria for lecture fees from AstraZeneca.

\section{Author details}

'Baylor University Medical Center, Texas Oncology and US Oncology, Dallas, TX, USA. ${ }^{2}$ Cancer Program at Sinai Health System, Toronto, Ontario, Canada. ${ }^{3}$ Ospedale San Gerardo Monza, Monza, Italy. ${ }^{4}$ AstraZeneca Pharmaceuticals, LP, Gaithersburg, MD, USA. ${ }^{5}$ AstraZeneca, Cambridge, UK. ${ }^{6} \mathrm{NHO}$ Shikoku Cancer Center, Matsuyama-shi, Ehime-Ken, Japan.

Received: 24 April 2020 Accepted: 5 October 2020

Published online: 27 October 2020

\section{References}

1. Bray F, Ferlay J, Soerjomataram I, Siegel RL, Torre LA, Jemal A. Global cancer statistics 2018: GLOBOCAN estimates of incidence and mortality worldwide for 36 cancers in 185 countries. CA Cancer J Clin. 2018;68(6):394-424.
2. Chen CC, Feng W, Lim PX, Kass EM, Jasin M. Homology-directed repair and the role of BRCA1, BRCA2, and related proteins in genome integrity and cancer. Annu Rev Cancer Biol. 2018;2:313-36.

3. Heeke AL, Pishvaian MJ, Lynce F, Xiu J, Brody JR, Chen W-J, Baker TM, Marshall $J$, Isaacs C. Prevalence of homologous recombination-related gene mutations across multiple cancer types. JCO Precis Oncol. 2018;2:1-13.

4. Ledermann JA, Drew Y, Kristeleit RS. Homologous recombination deficiency and ovarian cancer. Eur J Cancer. 2016;60:49-58.

5. O'Connor MJ. Targeting the DNA damage response in cancer. Mol Cell. 2015;60(4):547-60

6. Iqbal J, Ragone A, Lubinski J, Lynch HT, Moller P, Ghadirian P, Foulkes WD, Armel S, Eisen A, Neuhausen SL, et al. The incidence of pancreatic cancer in BRCA1 and BRCA2 mutation carriers. Br J Cancer. 2012;107(12):2005-9.

7. Moran A, O'Hara C, Khan S, Shack L, Woodward E, Maher ER, Lalloo F, Evans DG. Risk of cancer other than breast or ovarian in individuals with BRCA1 and BRCA2 mutations. Familial Cancer. 2012;11(2):235-42.

8. AstraZeneca. Lynparza significantly reduces the risk of disease worsening or death in patients with BRCA-mutated metastatic breast cancer [press release]. 2017. https://www.astrazeneca.com/media-centre/press-releases/2 017/lynparza-significantly-reduces-the-risk-of-disease-worsening-or-death-inpatients-with-BRCA-mutated-metastatic-breast-cancer-04062017.html. Accessed 26 Mar 2019

9. Tung NM, Robson ME, Ventz S, Santa-Maria CA, Marcom PK, Nanda R, Shah PD, Ballinger TJ, Yang ES, Melisko ME, et al. TBCRC 048: a phase II study of olaparib monotherapy in metastatic breast cancer patients with germline or somatic mutations in DNA damage response (DDR) pathway genes (Olaparib Expanded). J Clin Oncol. 2020;38.Abstract 1002.

10. Robson M, Im S-A, Senkus E, Xu B, Domchek SM, Masuda N, Delaloge S, Li W, Tung N, Armstrong A, et al. Olaparib for metastatic breast cancer in patients with a germline BRCA mutation. N Engl J Med. 2017;377(6):523-33.

11. Litton JK, Rugo HS, Ettl J, Hurvitz SA, Gonçalves A, Lee K-H, Fehrenbacher L, Yerushalmi R, Mina LA, Martin M, et al. Talazoparib in patients with advanced breast cancer and a germline BRCA mutation. N Engl J Med. 2018;379(8):753-63.

12. Domchek SM, Postel-Vinay S, Im S-A, Park YH, Delord J-P, Italiano A, Alexandre J, You B, Bastian S, Krebs MG, et al. Olaparib and durvalumab in patients with germline BRCA-mutated metastatic breast cancer (MEDIOLA): an open-label, multicentre, phase 1/2, basket study. Lancet Oncol. 2020; 21(9):1155-64

13. Abugattas J, Llacuachaqui M, Allende YS, Velásquez AA, Velarde R, Cotrina J, Garcés M, León M, Calderón G, de la Cruz M, et al. Prevalence of BRCA1 and BRCA2 mutations in unselected breast cancer patients from Peru. Clin Genet. 2015:88(4):371-5.

14. Gomes MC, Costa MM, Borojevic R, Monteiro AN, Vieira R, Koifman S, Koifman RJ, Li S, Royer R, Zhang S, et al. Prevalence of BRCA1 and BRCA2 mutations in breast cancer patients from Brazil. Breast Cancer Res Treat. 2007:103(3):349-53.

15. Hernandez JEL, Llacuachaqui M, Palacio GV, Figueroa JD, Madrid J, Lema M, Royer R, Li S, Larson G, Weitzel JN, et al. Prevalence of BRCA1 and BRCA2 mutations in unselected breast cancer patients from medellín, Colombia. Hered Cancer Clin Pract. 2014:12(1):11.

16. Høberg-Vetti H, Bjorvatn C, Fiane BE, Aas T, Woie K, Espelid H, Rusken T, Eikesdal HP, Listøl W, Haavind MT, et al. BRCA1/2 testing in newly diagnosed breast and ovarian cancer patients without prior genetic counselling: the DNA-BONus study. Eur J Hum Genet. 2016;24(6):881-8.

17. Li G, Guo X, Tang L, Chen M, Luo X, Peng L, Xu X, Wang S, Xiao Z, Yi W, et al. Analysis of BRCA1/2 mutation spectrum and prevalence in unselected Chinese breast cancer patients by next-generation sequencing. J Cancer Res Clin Oncol. 2017;143(10):2011-24.

18. Malone KE, Daling JR, Doody DR, Hsu L, Bernstein L, Coates RJ, Marchbanks PA, Simon MS, MCDonald JA, Norman SA, et al. Prevalence and predictors of BRCA1 and BRCA2 mutations in a population-based study of breast cancer in white and black American women ages 35 to 64 years. Cancer Res. 2006; 66(16):8297-308.

19. Kurian AW, Ward KC, Howlader N, Deapen D, Hamilton AS, Mariotto A, Miller D, Penberthy LS, Katz SJ. Genetic testing and results in a population-based cohort of breast cancer patients and ovarian cancer patients. J Clin Oncol. 2019;37(15):1305-15

20. Gelmon KA, Fasching PA, Couch F, Gelpi JB, Delaloge S, Labidi-Galy I, Bennett J, McCutcheon S, Ano S, O'Shaughnessy J. Real-world clinical effectiveness and safety of olaparib monotherapy in HER2-negative gBRCA- 
mutated metastatic breast cancer: phase IIIb LUCY interim analysis. J Clin Oncol 2020;38.Abstract 1087.

21. Bayraktar S, Gutierrez-Barrera AM, Lin H, Elsayegh N, Tasbas T, Litton JK, Ibrahim NK, Morrow PK, Green M, Valero V, et al. Outcome of metastatic breast cancer in selected women with or without deleterious BRCA mutations. Clin Exp Metastasis. 2013;30(5):631-42.

22. Daly MB, Pilarski R, Yurgelun MB, Berry MP, Buys SS, Dickson P, Domchek SM, Elkhanany A, Friedman S, Garber JE, et al. NCCN guidelines insights: genetic/familial high-risk assessment: breast, ovarian, and pancreatic, version 1.2020. J Natl Compr Cancer Netw. 2020;18(4):380-91.

23. Sharma P, Klemp JR, Kimler BF, Mahnken JD, Geier LJ, Khan QJ, Elia M, Connor CS, McGinness MK, Mammen JMW, et al. Germline BRCA mutation evaluation in a prospective triple-negative breast cancer registry: implications for hereditary breast and/or ovarian cancer syndrome testing. Breast Cancer Res Treat. 2014:145(3):707-14.

24. National Comprehensive Cancer Network. NCCN Clinical Practice Guidelines in Oncology - Breast Cancer v5.2020. 2020. https://www.nccn.org/ professionals/physician_gls/pdf/breast.pdf. Accessed 06 Aug 2020

25. The Cancer Genome Atlas Network. Comprehensive molecular portraits of human breast tumors. Nature. 2012;490(7418):61-70

26. Fasching PA, Hu C, Hart SN, Polley EC, Lee KY, Gnanolivu RD, Lilyquist J, Hartkopf AD, Taran FA, Janni W, et al. Cancer predisposition genes in metastatic breast cancer - association with metastatic pattern, prognosis, patient and tumor characteristics. Cancer Res. 2018;78.Abstract PD1-02.

27. Fasching PA, Brucker SY, Fehm TN, Overkamp F, Janni W, Wallwiener M, Hadji P, Belleville E, Häberle L, Taran F-A, et al. Biomarkers in patients with metastatic breast cancer and the PRAEGNANT study network. Geburtshilfe Frauenheilkd. 2015;75(1):41-50.

28. Zardavas D, Maetens M, Irrthum A, Goulioti T, Engelen K, Fumagalli D, Salgado R, Aftimos P, Saini KS, Sotiriou C, et al. The AURORA initiative for metastatic breast cancer. Br J Cancer. 2014;111(10):1881-7.

29. Abiltayeva A, Moore MA, Myssayev A, Adylkhanov T, Baissalbayeva A, Zhabagin K, Beysebayev E. Clinical, histopathological and molecular characteristics of metastatic breast cancer in North-Eastern Kazakhstan: a 10 year retrospective study. Asian Pac J Cancer Prev. 2016;17(10):4797-802.

30. Dawood S, Broglio K, Buzdar AU, Hortobagyi GN, Giordano SH. Prognosis of women with metastatic breast cancer by HER2 status and trastuzumab treatment: an institutional-based review. J Clin Oncol. 2010;28(1):92-8.

31. Gamucci T, Mentuccia L, Natoli C, Sperduti I, Cassano A, Michelotti A, Di Lauro L, Sergi D, Fabi A, Sarobba MG, et al. A real-world multicentre retrospective study of paclitaxel-bevacizumab and maintenance therapy as first-line for HER2-negative metastatic breast cancer. J Cell Physiol. 2017; 232(6):1571-8.

32. Gong C, Zhao Y, Wang B, Hu X, Wang Z, Zhang J, Zhang S. Efficacy and safety of everolimus in Chinese metastatic HR positive, HER2 negative breast cancer patients: a real-world retrospective study. Oncotarget. 2017; 8(35):59810-22.

33. Tiainen $\mathrm{L}$, Tanner $\mathrm{M}$, Lahdenperä $\mathrm{O}$, Vihinen $\mathrm{P}$, Jukkola A, Karihtala $\mathrm{P}$, Paunu $\mathrm{N}$, Huttunen T, Kellokumpu-Lehtinen PL. Bevacizumab combined with docetaxel or paclitaxel as first-line treatment of HER2-negative metastatic breast cancer. Anticancer Res. 2016;36(12):6431-8.

\section{Publisher's Note}

Springer Nature remains neutral with regard to jurisdictional claims in published maps and institutional affiliations.

\section{Ready to submit your research? Choose BMC and benefit from:}

- fast, convenient online submission

- thorough peer review by experienced researchers in your field

- rapid publication on acceptance

- support for research data, including large and complex data types

- gold Open Access which fosters wider collaboration and increased citations

- maximum visibility for your research: over $100 \mathrm{M}$ website views per year

At BMC, research is always in progress.

Learn more biomedcentral.com/submissions 